\title{
A Parametric Learning and Identification Based Robust Iterative Learning Control for Time Varying Delay Systems
}

\author{
Lun Zhai, Guohui Tian, and Yan Li \\ Service Robots Laboratory of Shandong University, School of Control Science and Engineering, Shandong University, \\ Shandong 250061, China \\ Correspondence should be addressed to Guohui Tian; g.h.tian@sdu.edu.cn
}

Received 9 July 2014; Accepted 4 September 2014; Published 23 September 2014

Academic Editor: Wuneng Zhou

Copyright (C) 2014 Lun Zhai et al. This is an open access article distributed under the Creative Commons Attribution License, which permits unrestricted use, distribution, and reproduction in any medium, provided the original work is properly cited.

A parametric learning based robust iterative learning control (ILC) scheme is applied to the time varying delay multiple-input and multiple-output (MIMO) linear systems. The convergence conditions are derived by using the $H_{\infty}$ and linear matrix inequality (LMI) approaches, and the convergence speed is analyzed as well. A practical identification strategy is applied to optimize the learning laws and to improve the robustness and performance of the control system. Numerical simulations are illustrated to validate the above concepts.

\section{Introduction}

Learning mechanism enables the human beings to master skills, while the experiences gained from practices play important roles in this procedure. It is expected that the learning mechanism can also be introduced to machines, which enables them to achieve satisfactory performance from previous acquired input-output information. The method of ILC was firstly applied to control manipulators at high speed which is proposed by Uchiyama [1]. In 1984, Arimoto [2] published the first English paper of ILC for accurate tracking of robot trajectories. The basic idea of ILC is utilizing the information of the previous iteration to realize perfect tracking without exact knowledge of the system parameters, and a typical ILC scheme is shown as in Figure 1. In the recent three decades, many kinds of learning laws are utilized which can be mainly divided by two categories: the linear learning laws and the nonlinear learning laws. For example, the linear learning laws include but are not limited to the parametric learning law $[3,4]$, the robust learning law [5], the highorder learning law $[6,7]$, the PD type learning law $[8,9]$, and so on [10]. On the other hand, the Newton learning law and the Secant learning law belong to the nonlinear ones $[11,12]$ which have faster convergence speed comparing to some linear cases. Moreover, the control objectives are mainly focused on the linear continuous and discrete forms $[13,14]$ and the nonlinear systems with relative degree one [15] or the quasilinear forms [16] and so forth [17-27].

The time delay systems are ubiquitous in real world control problems [28] such as networked control systems, chemical processes, hydraulic, and rolling mill systems. The time delay affects the system performance in a large scale. Serious performance degradation and even instability can be led by time delay [29]. For decades, considerable efforts have been paid to assure the robust performance of time delay systems in both theories and applications [30]. The research of time delayed system is usually divided into two categories: the constant time delays and the time varying delays. Meanwhile, the time delayed systems can be divided into delay dependent (the case in the paper) and delay independent cases [31]. In real projects, some typical existences of time delay are illustrated in Figure 2. Because of the time delay, the analysis of the convergence conditions and the convergence speed is more complicated. Although the small learning gains can guarantee the convergence, they sacrifice the convergence speed accordingly. A practical identification strategy is urgently required along with the iterative learning procedures.

In fact, the ILC scheme is an inverse solution of control systems. Based on this fact, the input and output data are 


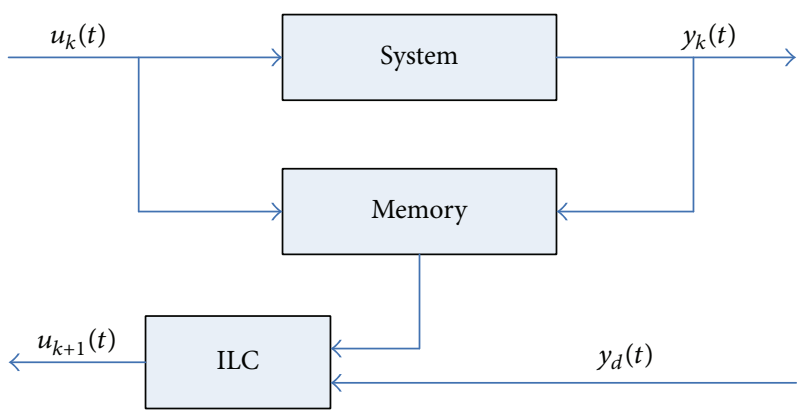

FIgURE 1: The basic idea of ILC.

the most reliable source for the identification method. That is the reason why the ILC scheme can be coordinated with the identification method. The system identifications are mainly dedicated to the identifications of the structure uncertainties and the parametric uncertainties [32-35]. In most ILC schemes, the system structure is known in advance, which exactly meets the basic requirements of system identification. The final identification result is expected to achieve the same output data as the real system [36]. Many researches focus on various identification methods and their applications in these years [37-42]. Particularly, the data driven ILC becomes a hotspot nowadays [43]. Although the aforementioned researches have gained great harvest, to the best of our knowledge, the analysis of time varying delay system with parametric learning law by supplement of identification remains open. Besides, it is a meaningful work to combine system identification and ILC together so that they can benefit from each other. Different identified models can be gained by each group of the data information. The optimal solution can be gained from the identified models by the minimum average distance method so that the optimal controller can be designed by the parameters of the optimal identified models. The faster convergence speed can be expected and the complexity of controller design is reduced efficiently.

In this paper, a parametric robust ILC scheme is discussed. The feedback part [44] in the controller is designed by using the current tracking error which improves the robustness of the system, and the feedforward control process is a PD type controller. By applying the similar techniques in $H_{\infty}$ method $[45,46]$ to the time varying delayed system, the convergence conditions are converted into linear matrix inequality (LMI) forms [47]. To the constant time delay systems the convergence conditions in the form of $\infty$-norm by the contraction mapping method are achieved, and the convergence speed is analyzed. Lastly, a practical identification strategy is applied to the optimization of learning laws so that the control performance is improved to a great extent.

The following paper is organized as follows: the problem statement is formulated in Section 2. In Section 3 the convergence condition and some correlated results are analyzed. A practical identification strategy is shown in Section 4. In Section 5, a number of numerical simulations are applied to validate above discussions. Lastly, some concluding remarks are summarized in Section 6.

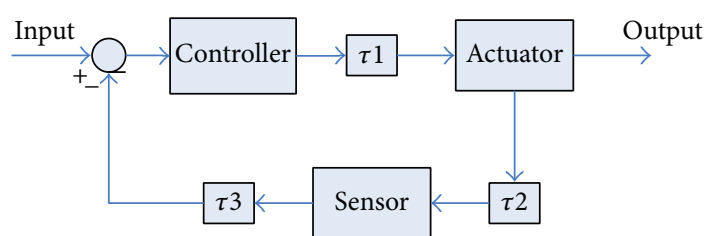

Figure 2: A control process with various time delays.

\section{Problem Formulation}

In this section, the problem of convergence analysis of the time varying delayed system is formulated and some preliminary knowledge is achieved. The LMI approach is applied to gain the convergence condition, in which the problem is transferred to be a standard $H_{\infty}$ problem and the Schur complement is introduced in the end of this section.

Consider the multiple-input multiple-output time varying delay system

$$
\begin{aligned}
& \dot{x}_{k}(t)=A x_{k}(t)+A_{h} x_{k}\left(t-d_{k}(t)\right)+B u(t), \\
& y_{k}(t)=C x_{k}(t)
\end{aligned}
$$

where $k, x_{k}(t), y_{k}(t)$, and $u_{k}(t)$ are, respectively, the iteration number, state, output, and input of the system, while $A, A_{h}$, $B$, and $C$ are the parameters of the system with appropriate dimensions. The time delay considered in the paper exists in the process between the controller and the actuator as one of the cases shown in Figure 2. The time delay in the state is denoted by $d_{k}(t) \in[0, T]$. The time varying delay is nonrepeatable. The exact value of time delay is not needed to be known while only the information including the bound of time delay and that of the derivative of time delay is necessary. The output tracking error is described by $e_{k}(t)=y_{d}(t)-y_{k}(t)$.

The ILC scheme uses the tracking error in the parametric learning law [3] which is shown as

$$
\begin{aligned}
& u_{k}(t)=K_{1} e_{k}(t)+\theta_{k}(t) \\
& \theta_{k}(t)=\theta_{k-1}(t)+K_{2} \dot{e}_{k-1}(t)+K_{3} e_{k-1}(t)
\end{aligned}
$$

It is obvious from the above equation that the input is influenced by the current and the previous information, and the learning law of parameter $\theta_{k}(t)$ is the PD type as shown in Figure 3. The transfer function of the system is $G_{p}(t)$, where $y_{k}(t)=G_{p}(t) x_{k}(t)$ and another transfer function is $G_{e k}(t)$ which is the transfer function between $e_{k}(t)$ and $e_{k-1}(t)$, and the monotonic convergence condition can be achieved by the constriction that $\left\|G_{e k}(t)\right\|_{\infty}<1$, where $e_{k}(t)=G_{e k}(t) e_{k-1}(t)$.

However, this is not a standard $H_{\infty}$ control problem while there is no disturbance in the system. Apply $\varepsilon_{1}, \varepsilon_{2}$, and $\widehat{G}_{e k}$ to convert the above problem to a standard one [48] as below. Define $\gamma=\varepsilon_{1} \varepsilon_{2}^{-1}$ so that

$$
\left\|\widehat{G}_{e k}\right\|_{\infty}<\varepsilon_{1},
$$




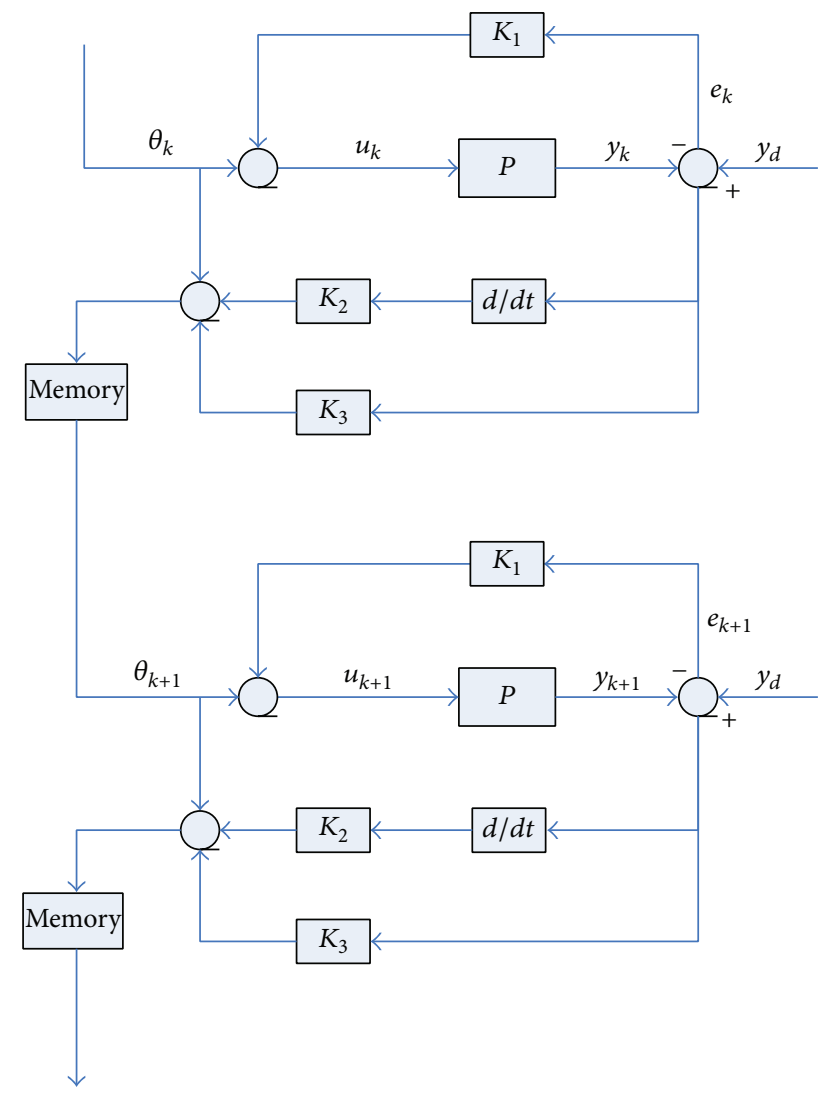

FIGURE 3: The parametric learning ILC process for the time varying delay systems.

which can be considered as a standard $H_{\infty}$ control problem. Here $\varepsilon_{1} \leq \varepsilon_{2}, \widehat{G}_{e k}=\varepsilon_{2} G_{e k}$. If the condition above is satisfied, the conclusion that the output tracking error converges monotonically to zero is achieved as the iteration $k$ increases to infinity.

It is known that if the time delay is time invariant, the convergent analysis is convenient in the frequency domain by applying Laplace transform and the contraction mapping method $[49,50]$. However, the time delay here is time varying so that the convergent analysis is more complicated. To overcome the difficulties brought by the time varying delay, the LMI method is applied to gain the convergence condition and the controller can be designed to be the optimal one by the solutions of the LMIs.

Then another transformation $T_{e k}=\widehat{G}_{e k}^{T}$ is introduced; at the same time, $X_{2}=-\varepsilon_{2} K_{2}$ and $X_{3}=-\varepsilon_{2} K_{3}$ are defined. So $T_{e k}$ could be viewed as the transformation function between $z(t)$ and $w(t)$ of the following system which is

$$
\begin{aligned}
& \dot{x}_{k}(t)=\widehat{A} x_{k}(t)+\widehat{A}_{h} x_{k}\left(t-d_{k}(t)\right)+\widehat{B} \omega_{k}(t), \\
& z_{k}(t)=\widehat{C} x_{k}(t)+\widehat{C}_{h} x_{k}\left(t-d_{k}(t)\right)+\widehat{D} \omega_{k}(t),
\end{aligned}
$$

where $z(t)$ is the output of the transformed system and $w(t)$ can seem as a kind of disturbance, and the time varying delay is differential and satisfies

$$
d_{k}(t) \leq h<\infty, \quad \dot{d}_{k}(t) \leq \mu<1,
$$

and the parameters of the system are

$$
\begin{aligned}
\widehat{A} & =\left(A-B K_{1} C\right)^{T}, \\
\widehat{A}_{h} & =A_{h}^{T}, \\
\widehat{B} & =C^{T}, \\
\widehat{C} & =\left(\left(A-B K_{1} C\right) B X_{2}+B X_{3}\right)^{T}, \\
\widehat{C}_{h} & =\left(A_{h} B X_{2}\right)^{T}, \\
\widehat{D} & =\left(\varepsilon_{2} I+C B X_{2}\right)^{T} .
\end{aligned}
$$

Moreover, a relevant lemma which is called Schur complement is introduced for further usage.

Lemma 1 (Schur complement). For a given symmetric matrix

$$
S=S^{T}=\left(\begin{array}{cc}
S_{11} & S_{12} \\
* & S_{22}
\end{array}\right),
$$

where $S_{11} \in \mathbb{R}^{r \times r}$, the following conditions are equivalent [51]:

(1) $S<0$;

(2) $S_{11}<0, S_{22}-S_{12}^{T} S_{11}^{-1} S_{12}<0$ or $S_{22}<0, S_{11}-S_{12} S_{22}^{-1} S_{12}^{T}<$ 0 .

Assumption 2. The assumption that the system is controllable and observable is satisfied. For a NCS, if the system is controllable and observable, that means we can determine a controller that will stabilize the system using only input and output measurements. It is shown that $C$ is full column rank because the system is observable while $B$ is full column rank because the system is controllable. Then system (1) can be described by the relationships between the input and the output as below:

$$
\begin{aligned}
\dot{y}_{k}(t)= & C A\left(C^{T} C\right)^{-1} C^{T} y_{k}(t) \\
& +C A_{h}\left(C^{T} C\right)^{-1} C^{T} y_{k}(t-h)+C B u_{k}(t) .
\end{aligned}
$$

\section{Convergence Condition}

The monotonic convergence condition in the form of LMIs is achieved in this section. By solving the LMIs, the formula of the optimal learning gains can be constructed accordingly. The time delayed system with the ILC scheme and the time varying delay system are considered as well.

Theorem 3. Consider the time varying delay system as

$$
\begin{aligned}
& \dot{x}_{k}(t)=A x_{k}(t)+A_{h} x_{k}\left(t-d_{k}(t)\right)+B u_{k}(t), \\
& y_{k}(t)=C x_{k}(t),
\end{aligned}
$$

with the parametric learning law which is shown as (2). The tracking error of the system converges to zero monotonically as the iteration increases if the following LMIs are satisfied. There 
exist $Y>0, P>0, Q>0, Z>0$, and any matrix $N_{1}, N_{2}, X_{2}$, or $X_{3}$ with proper dimensions such that

$$
\begin{aligned}
\widehat{\Delta} & =\left(\begin{array}{ccccc}
-\varepsilon_{1} I & \widehat{C} & \widehat{D} & \widehat{C}_{h} & 0 \\
* & \widehat{\Phi}_{11} & 0 & \widehat{\Phi}_{12} & h \widehat{A}^{T} \widehat{Z} \\
* & * & -\varepsilon_{1} I & 0 & 0 \\
* & * & * & \widehat{\Phi}_{22} & h \widehat{A}_{h}^{T} \widehat{Z} \\
* & * & * & * & -h \widehat{Z}
\end{array}\right)<0, \\
\Pi & =\left(\begin{array}{ccc}
h Y 11 & h Y 12 & N_{1} \\
* & h Y 22 & N_{2} \\
* & * & Z
\end{array}\right)>0,
\end{aligned}
$$

where the parameters are shown in (6).

Proof. For achieving better system performance, the stability of the system should be satisfied [48]. In this part, a Lyapunov function candidate [51] is applied to derive the stability of (9). The time delay system is considered as

$$
\begin{aligned}
& \dot{x}_{k}(t)=\widehat{A} x_{k}(t)+\widehat{A}_{h} x_{k}\left(t-d_{k}(t)\right), \\
& x_{k}(t)=0, \quad t \in(0, h),
\end{aligned}
$$

where

$$
\begin{gathered}
0<d_{k}(t) \leq h<\infty, \\
\dot{d}_{k}(t) \leq \mu<1 .
\end{gathered}
$$

The derivative of time delay is not restricted to $\dot{d}_{k}(t)>0$ for the reason that the negative $\dot{d}_{k}(t)$ means the time delay will decrease to zero as the iteration number $k$ increases. So only the upper bound of the $\dot{d}_{k}(t)$ is restricted.

A Lyapunov function candidate is defined as

$$
\begin{aligned}
V_{k}(t)= & y_{k}^{T}(t) \widetilde{P} y_{k}(t)+\int_{t-d_{k}(t)}^{t} y_{k}^{T}(s) \widetilde{Q} y_{k}(s) d s \\
& +\int_{-h}^{0} \int_{t+\theta}^{t} \dot{y}_{k}^{T}(s) \widetilde{Z} \dot{y}_{k}(s) d s d \theta,
\end{aligned}
$$

where $y_{k}(t)$ is the measurable system out in the $k$ th iteration and the matrices $\widetilde{P}, \widetilde{Q}$, and $\widetilde{Z}$ satisfy the constriction that $\widetilde{P}>$ $0, \widetilde{Q}>0$, and $\widetilde{Z}>0$ which make $V_{k}(t)>0$. Define $P=C^{T} \widetilde{P} C$, $Q=C^{T} \widetilde{Q} C$, and $Z=C^{T} \widetilde{Z} C$; the first order derivative of $V_{k}(t)$ is

$$
\begin{aligned}
\dot{V}_{k}(t)= & \dot{x}_{k}^{T}(t) P x_{k}(t)+x_{k}^{T}(t) P \dot{x}_{k}(t)+x_{k}^{T}(t) Q x_{k}(t) \\
& -\left(1-\dot{d}_{k}(t)\right) x_{k}^{T}\left(t-d_{k}(t)\right) Q x_{k}\left(t-d_{k}(t)\right) \\
& +h \dot{x}_{k}^{T}(t) Z \dot{x}_{k}(t)-\int_{t-h}^{t} \dot{x}_{k}^{T}(s) Z \dot{x}_{k}(s) d s
\end{aligned}
$$

$$
\begin{aligned}
\leq & \dot{x}_{k}^{T}(t) P x_{k}(t)+x_{k}^{T}(t) P \dot{x}_{k}(t)+x_{k}^{T}(t) Q x_{k}(t) \\
& -\left(1-\dot{d}_{k}(t)\right) x_{k}^{T}\left(t-d_{k}(t)\right) Q x_{k}\left(t-d_{k}(t)\right) \\
& +h \dot{x}_{k}^{T}(t) Z \dot{x}_{k}(t)-\int_{t-h}^{t} \dot{x}_{k}^{T}(s) Z \dot{x}_{k}(s) d s \\
& +2\left[x_{k}^{T}(t) N_{1}+x_{k}^{T}\left(t-d_{k}(t)\right) N_{2}\right] \\
& \times\left[x_{k}(t)-\int_{t-d_{k}(t)}^{t} \dot{x}_{k}(s) d s-x_{k}\left(t-d_{k}(t)\right)\right] \\
& +h \eta_{1}^{T}(t) Y \eta_{1}(t)-\int_{t-d_{k}(t)}^{t} \eta_{1}^{T}(t) Y \eta_{1}(t) d s \\
= & \eta_{1}^{T}(t) \Xi \eta_{1}(t)-\int_{t-d_{k}(t)}^{t} \eta_{2}^{T}(t, s) \Psi \eta_{2}(t, s) d s,
\end{aligned}
$$

where $N_{1}$ and $N_{2}$ are appropriately dimensioned matrices and

$$
Y=\left(\begin{array}{cc}
Y 11 & Y 12 \\
* & Y 22
\end{array}\right)
$$

From (9) and (2), the following inequality is achieved:

$$
\begin{aligned}
\dot{V}_{k}(t) \leq & {\left[\widehat{A} x_{k}(t)+\widehat{A}_{h} x_{k}\left(t-d_{k}(t)\right)\right]^{T} P x_{k}(t) } \\
& +x_{k}^{T}(t) P\left[\widehat{A} x_{k}(t)+\widehat{A}_{h} x_{k}\left(t-d_{k}(t)\right)\right] \\
& +x_{k}^{T}(t) Q x_{k}(t) \\
& -(1-\mu) x_{k}^{T}\left(t-d_{k}(t)\right) Q x_{k}\left(t-d_{k}(t)\right) \\
& +h\left[\widehat{A} x_{k}(t)+\widehat{A}_{h} x_{k}\left(t-d_{k}(t)\right)\right]^{T} \\
& \times Z\left[\widehat{A} x_{k}(t)+\widehat{A}_{h} x_{k}\left(t-d_{k}(t)\right)\right] \\
& -\int_{t-h}^{t} \dot{x}_{k}^{T}(s) Z \dot{x}_{k}(s) d s \\
& +2\left[x_{k}^{T}(t) N_{1}+x_{k}^{T}\left(t-d_{k}(t)\right) N_{2}\right] \\
& \times\left[x_{k}(t)-\int_{t-d_{k}(t)}^{t} \dot{x}_{k}(s) d s-x_{k}\left(t-d_{k}(t)\right)\right] \\
& +h \eta_{1}^{T}(t) Y \eta_{1}(t)-\int_{t-d_{k}(t)}^{t} \eta_{1}^{T}(t) Y \eta_{1}(t) d s \\
= & \eta_{1}^{T}(t) \Xi \eta_{1}(t)-\int_{t-d_{k}(t)}^{t} \eta_{2}^{T}(t, s) \Psi \eta_{2}(t, s) d s,
\end{aligned}
$$

where

$$
\begin{aligned}
& \eta_{1}(t)=\left[x_{k}^{T}(t), x_{k}^{T}\left(t-d_{k}(t)\right)\right]^{T}, \\
& \eta_{2}(t)=\left[x_{k}^{T}(t), x_{k}^{T}\left(t-d_{k}(t)\right), \dot{x}_{k}^{T}(s)\right]^{T} .
\end{aligned}
$$


The matrices $\Xi$ and $\Psi$ equal

$$
\begin{aligned}
& \Xi=\left(\begin{array}{cc}
\xi_{11} & \xi_{12} \\
* & \xi_{22}
\end{array}\right), \\
& \Psi=\left(\begin{array}{ccc}
h Y 11 & h Y 12 & N_{1} \\
* & h Y 22 & N_{2} \\
* & * & Z
\end{array}\right),
\end{aligned}
$$

where

$$
\begin{aligned}
\xi_{11}= & \widehat{A}^{T} P+P \widehat{A}+Q+h \widehat{A}^{T} Z \widehat{A} \\
& +N_{1}^{T}+N_{1}+h Y_{11} \\
\xi_{12}= & P \widehat{A}_{h}+h \widehat{A}^{T} Z \widehat{A}_{h}-N_{1}+N_{2}^{T}+h Y_{12} \\
\xi_{22}= & -(1-\mu) Q+h \widehat{A}_{h}^{T} Z \widehat{A}_{h}-N_{2}-N_{2}^{T}+h Y_{22} .
\end{aligned}
$$

Thus the equilibrium of system (9) is asymptotically stable if $\dot{V}_{k}(t)<0$; that is, the following inequalities are satisfied [51, 52]:

$$
\begin{aligned}
& \Xi<0, \\
& \Psi>0 .
\end{aligned}
$$

Then a performance index $J$ is applied so that $\left\|G_{e}(t)\right\|_{\infty}<1$ if $J<0$ :

$$
J=\int_{0}^{t} z_{k}^{T}(t) z_{k}(t)-\varepsilon_{1}^{2} \omega_{k}^{T}(t) \omega_{k}(t) d t
$$

The Lyapunov based approach is applied to deduce that

$$
\begin{aligned}
J & =\int_{0}^{t} z_{k}^{T}(t) z_{k}(t)-\varepsilon_{2}^{2} \omega_{k}^{T}(t) \omega_{k}(t) d t \\
< & \int_{0}^{t} z_{k}^{T}(t) z_{k}(t)-\varepsilon_{2}^{2} \omega_{k}^{T}(t) \omega_{k}(t) d t+V_{k}(t) \\
& =\int_{0}^{t} z_{k}^{T}(t) z_{k}(t)-\varepsilon_{2}^{2} \omega_{k}^{T}(t) \omega_{k}(t)+\dot{V}_{k}(t) d t \\
& =\int_{0}^{t}\left[\widehat{C} x_{k}(t)+\widehat{C}_{h} x_{k}(t-h)+\widehat{D} \omega_{k}(t)\right]^{T} \\
& \times\left[\widehat{C} x_{k}(t)+\widehat{C}_{h} x_{k}(t-h)+\widehat{D} \omega_{k}(t)\right] \\
& -\int_{0}^{t} \omega_{k}^{T}(t) \omega_{k}(t)+\dot{V}_{k}(t) d t \\
& -\int_{0}^{T}(t) \Pi \eta_{3}(t) d t \\
& \int_{t-d_{k}(t)}^{t} \eta_{4}^{T}(t, s) \Omega \eta_{4}(t, s) d s d t
\end{aligned}
$$

where

$$
\begin{aligned}
\eta_{3}(t) & =\left[x_{k}^{T}(t), \omega_{k}^{T}(t), x_{k}^{T}\left(t-d_{k}(t)\right)\right]^{T}, \\
\eta_{4}(t) & =\left[x_{k}^{T}(t), x_{k}^{T}\left(t-d_{k}(t)\right), \dot{x}_{k}^{T}(s)\right]^{T}, \\
\Pi & =\left(\begin{array}{ccc}
\pi_{11} & \pi_{12} & \pi_{13} \\
* & \pi_{22} & \pi_{23} \\
* & * & \pi_{33}
\end{array}\right),
\end{aligned}
$$

$$
\Omega=\left(\begin{array}{ccc}
h Y_{11} & h Y_{12} & N_{1} \\
* & h Y_{22} & N_{2} \\
* & * & Z
\end{array}\right) .
$$

The components of $\Pi$ are detailed as

$$
\begin{aligned}
& \pi_{11}=\Phi_{11}+h \widehat{A}^{T} Z \widehat{A}^{T}+\widehat{C}^{T} \widehat{C}, \\
& \pi_{12}=\widehat{C}^{T} \widehat{D}, \\
& \pi_{13}=\Phi_{13}+h \widehat{A}^{T} Z \widehat{A}_{h}+\widehat{C}^{T} \widehat{C}_{h}, \\
& \pi_{22}=-\varepsilon_{1}^{2}+\widehat{D}^{T} \widehat{D}, \\
& \pi_{23}=\widehat{D}^{T} \widehat{C}_{h}, \\
& \pi_{33}=\Phi_{33}+h \widehat{A}_{h}^{T} Z \widehat{A}_{h}+\widehat{C}_{h}^{T} \widehat{C}_{h},
\end{aligned}
$$

where

$$
\begin{aligned}
& \Phi_{11}=\widehat{A}^{T} P+P \widehat{A}+Q+N_{1}^{T}+N_{1}+h Y_{11}, \\
& \Phi_{12}=P \widehat{A}_{h}-N_{1}+N_{2}^{T}+h Y_{12} \\
& \Phi_{22}=-(1-\mu) Q-N_{2}-N_{2}^{T}+h Y_{22} .
\end{aligned}
$$

The matrices $\Pi<0$ and $\Omega>0$ guarantee $J<0$, which means that $\|z\|_{2}<\varepsilon_{1}\|\omega\|_{2}$; that is, $\|T e\|_{\infty}<\varepsilon_{1}$. Then it is known that if the above conditions are satisfied, system (1) with the parametric learning law (2) is monotonically convergent. Applying the Schur complement to $\Pi<0$ by two times yields $\Delta<0$, where

$$
\Delta=\left(\begin{array}{ccccc}
-\varepsilon_{1} I & \widehat{C} & \widehat{D} & \widehat{C}_{h} & 0 \\
* & \Phi_{11} & 0 & \Phi_{12} & h \widehat{A}^{T} Z \\
* & * & -\varepsilon_{1}^{2} & 0 & 0 \\
* & * & * & \Phi_{22} & h \widehat{A}_{h}^{T} Z \\
* & * & * & * & -h Z
\end{array}\right)
$$

Pre- and postmultiplying $\Delta$ by the following diagonal matrix yields

$$
\left(\begin{array}{ccccc}
\varepsilon_{1}^{1 / 2} & 0 & 0 & 0 & 0 \\
0 & \varepsilon_{1}^{-1 / 2} & 0 & 0 & 0 \\
0 & 0 & \varepsilon_{1}^{-1 / 2} & 0 & 0 \\
0 & 0 & 0 & \varepsilon_{1}^{-1 / 2} & 0 \\
0 & 0 & 0 & 0 & \varepsilon_{1}^{-1 / 2}
\end{array}\right) .
$$


Define

$$
\begin{array}{cc}
\widehat{\Phi}_{11}=\varepsilon_{1}^{-1} \Phi_{11}, & \widehat{\Phi}_{12}=\varepsilon_{1}^{-1} \Phi_{12}, \\
\widehat{\Phi}_{22}=\varepsilon_{1}^{-1} \Phi_{22}, & \widehat{P}=\varepsilon_{1}^{-1} P, \\
\widehat{P}=\varepsilon_{1}^{-1} P, & \widehat{Q}=\varepsilon_{1}^{-1} Q, \\
\widehat{Z}=\varepsilon_{1}^{-1} Z, & \widehat{N}_{1}=\varepsilon_{1}^{-1} N_{1}, \\
\widehat{N}_{2}=\varepsilon_{1}^{-1} N_{2}, & \widehat{Y}_{11}=\varepsilon_{1}^{-1} Y_{11}, \\
\widehat{Y}_{12}=\varepsilon_{1}^{-1} Y_{12}, & \widehat{Y}_{22}=\varepsilon_{1}^{-1} Y_{22} ;
\end{array}
$$

we have the following LMI:

$$
\widehat{\Delta}=\left(\begin{array}{ccccc}
-\varepsilon_{1} I & \widehat{C} & \widehat{D} & \widehat{C}_{h} & 0 \\
* & \widehat{\Phi}_{11} & 0 & \widehat{\Phi}_{12} & h \widehat{A}^{T} \widehat{Z} \\
* & * & -\varepsilon_{1} I & 0 & 0 \\
* & * & * & \widehat{\Phi}_{22} & h \widehat{A}_{h}^{T} \widehat{Z} \\
* & * & * & * & -h \widehat{Z}
\end{array}\right)<0
$$

where

$$
\begin{aligned}
& \widehat{\Phi}_{11}=\widehat{A}^{T} \widehat{P}+\widehat{P} \widehat{A}+\widehat{Q}+\widehat{N}_{1}^{T}+\widehat{N}_{1}+h \widehat{Y}_{11}, \\
& \widehat{\Phi}_{12}=\widehat{P} \widehat{A}_{h}-\widehat{N}_{1}+\widehat{N}_{2}^{T}+h \widehat{Y}_{12}, \\
& \widehat{\Phi}_{22}=-(1-\mu) \widehat{Q}-\widehat{N}_{2}-\widehat{N}_{2}^{T}+h \widehat{Y}_{22} .
\end{aligned}
$$

Here ends the proof.

Remark 4. The basic idea of ILC scheme is to improve the performance of a system only by using the input and output data. The characteristic of the ILC is an adaptive control process which means that the precise system parameters are not necessary to be known. However, by improving the complexity of the system such as the introduction of time delay, package dropout, and structure uncertainties, the traditional ILC needs the support of identification methods to optimize the convergence condition with more known information, especially some key information for the analysis.

\section{System Identification and Optimization}

The increase of complexity of convergence condition requires more accurate parameter information so that an optimized learning gain can be designed, and a faster convergence speed can be expected as well. In this section, a practical continuous-time system identification method is discussed to determine the unknown parameters, which is further applied to optimize the learning law (2). On the other hand, the identification results are improved along with the iterative processes [41]. It should be noted that, in the ILC scheme, the system structure is determined in advance, which fully agrees with the requirement of system identification. Besides, the ILC scheme (2) considers only the input-output of (1), and the system identification is aiming at the search of a model by using the input-output data. Thus, in this section, a timeinvariant model is identified iteratively and the identified parameters are applied to the optimal design of control laws, where the feedback term improves the robustness to the uncertainties.

Consider the time delay scalar system

$$
\begin{aligned}
& \dot{x}_{k}(t)=A x_{k}(t)+A_{h} x_{k}(t-h)+B u_{k}(t), \\
& y_{k}(t)=C x_{k}(t) .
\end{aligned}
$$

In the frequency domain, the transfer function is

$$
G_{p}(s)=\frac{Y(s)}{U(s)}=\frac{C B}{s I-A-A_{h} e^{-h s}}
$$

thus

$$
\begin{aligned}
Y(s) & =\frac{1}{A} s Y(s)-\frac{A_{h}}{A} e^{-h s} Y(s)-\frac{C B}{A} U(s) \\
& =\left(\begin{array}{lll}
s Y(s)-e^{-h s} Y(s) & -U(s)
\end{array}\right)\left(\begin{array}{c}
\frac{1}{A} \\
\frac{A_{h}}{A} \\
\frac{C B}{A}
\end{array}\right) .
\end{aligned}
$$

Applying the inverse Laplace transform to the above equation yields

$$
\begin{aligned}
& y(t)=\frac{1}{A} y^{\prime}(t)-\frac{A_{h}}{A} y(t-h)-\frac{C B}{A} u(t)
\end{aligned}
$$

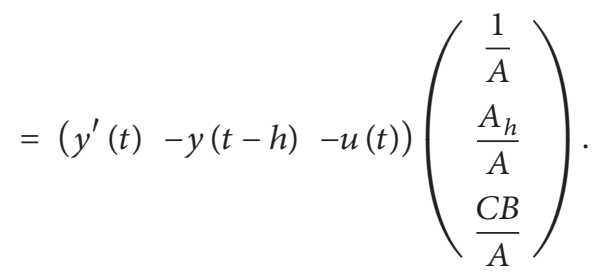

Then the parameters of the system can be achieved by the data of system inputs and outputs. Let

$$
\Lambda(t)=\left(y^{\prime}(t)-y(t-h)-u(t)\right)
$$

and let $t_{1}, t_{2}, t_{3}, \ldots, t_{n}$ be different sample time instants; it follows that

$$
\begin{aligned}
& \left(\begin{array}{lllll}
y\left(t_{1}\right) & y\left(t_{2}\right) & y\left(t_{3}\right) & \cdots & y\left(t_{n}\right)
\end{array}\right)^{T}
\end{aligned}
$$

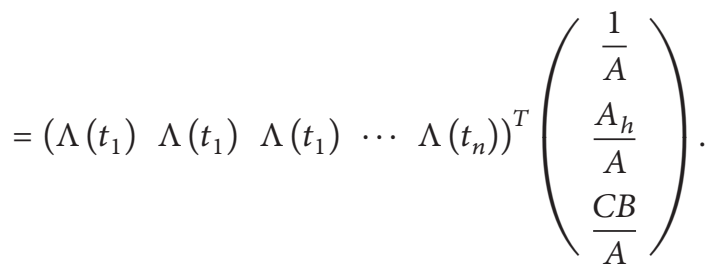


Define

$$
\begin{aligned}
& \check{Y}=\left(\begin{array}{lllll}
y\left(t_{1}\right) & y\left(t_{2}\right) & y\left(t_{3}\right) & \cdots & y\left(t_{n}\right)
\end{array}\right)^{T}, \\
& \check{\Lambda}=\left(\Lambda\left(t_{1}\right) \Lambda\left(t_{1}\right) \Lambda\left(t_{1}\right) \cdots \Lambda\left(t_{n}\right)\right)^{T}, \\
& \check{\Theta}=\left(\begin{array}{c}
\frac{1}{A} \\
\frac{A_{h}}{A} \\
\frac{C B}{A}
\end{array}\right) ;
\end{aligned}
$$

it follows that

$$
\check{Y}=\check{\Lambda} \check{\Theta} .
$$

The matrix $\Lambda$ has full column rank so that $\check{\Lambda}$ is also a full column rank matrix. Based on the above relationships, the following equation is achieved:

$$
\left(\check{\Lambda}^{T} \check{\Lambda}\right)^{-1} \check{\Lambda}^{T} \check{Y}=\check{\Theta}
$$

Then the unknown parameters of the system can be estimated by using (39) iteratively [36].

Remark 5. Only the information of system structure is utilized in both the processes of ILC and identification. The solutions by the identification method can optimize the controller of the time varying delay system. A group of system parameters are obtained by a group of iterations of control processes so that $k$ groups of system parameters can be achieved by $k$ steps of control iterations. The optimal parameters are the key factors of the optimal learning laws, which is derived from the minimum average distance method. The solutions of the identified parameters can be shown in an $n$-dimensional Cartesian coordinate, where $d_{m, n}$ denotes the distance of the $m$ th and $n$th groups of identified parameters. The optimal identification result is

$$
\check{\Theta}_{\text {optimal }}=\left\{\check{\Theta}_{m} \mid \min _{m} \frac{\sum_{n=1}^{k} d_{m, n}}{k}\right\} .
$$

\section{Illustrated Examples}

5.1. Example 1. The convergence condition by LMIs is simulated in this subsection. The time delay system (1) is considered with the following parameters in the form of matrix:

$$
\begin{aligned}
A & =\left(\begin{array}{ccc}
-2.1 & 2.2 & 0.2 \\
-1.6 & -1.1 & 1.8 \\
-0.2 & 0.6 & -1.3
\end{array}\right), \\
A_{h} & =\left(\begin{array}{ccc}
-0.4 & -0.8 & 0.4 \\
0.3 & -1.1 & -1.5 \\
0.5 & -0.3 & -0.5
\end{array}\right),
\end{aligned}
$$

$$
\begin{aligned}
& B=\left(\begin{array}{cc}
0.2 & 0.8 \\
-0.6 & 0.2 \\
-1.2 & 1.0
\end{array}\right), \\
& C=\left(\begin{array}{ccc}
0.3 & 0.5 & -1.7 \\
0.3 & 0.1 & 0.3
\end{array}\right),
\end{aligned}
$$

and the learning gain $K_{1}$ in the learning law (2) equals

$$
K_{1}=\left(\begin{array}{ll}
0.3 & 0.4 \\
0.5 & 0.8
\end{array}\right)
$$

The upper bound of the time varying delay is $h=0.1$, and the derivative of $d_{k}(t)$ is 0.1 . Through MATLAB/LMI the feasibility conclusion that $t=-0.0664$ is achieved which means that the monotonical convergence conditions in the form of LMI in Theorem 3 are satisfied. And the best results which lead to the least conservation are

$$
\begin{aligned}
P & =\left(\begin{array}{ccc}
13.6287 & 2.7458 & 1.4555 \\
2.7458 & 10.5883 & -1.1852 \\
1.4555 & -1.1852 & 6.9604
\end{array}\right), \\
Q & =\left(\begin{array}{ccc}
14.4263 & 0.9467 & 2.5456 \\
0.9467 & 15.4343 & -4.6042 \\
2.5456 & -4.6042 & 9.4211
\end{array}\right), \\
Z & =\left(\begin{array}{ccc}
14.9660 & 4.1603 & 4.2553 \\
4.1603 & 18.6389 & 3.1268 \\
4.2553 & 3.1268 & 14.1099
\end{array}\right) .
\end{aligned}
$$

The other learning gains $K_{2}$ and $K_{3}$ which are the gains of the optimal controller are also gained by the solution of $\varepsilon_{2}, X_{2}$, and $X_{3}$ which is

$$
\begin{aligned}
K_{2} & =-\varepsilon_{2}^{-1} X_{2} \\
& =\left(\begin{array}{ll}
0.1878 & 0.0536 \\
0.0536 & 0.1684
\end{array}\right), \\
K_{3} & =-\varepsilon_{2}^{-1} X_{3} \\
& =\left(\begin{array}{ll}
0.0619 & 0.1713 \\
0.1713 & 0.4025
\end{array}\right) .
\end{aligned}
$$

By the least conservatism solutions of the LMI, the Lyapunov function is achieved and the learning gains can be designed to be the gains of the optimal controller which can improve the system performance.

\subsection{Example 2. Let the system be}

$$
\begin{aligned}
& \dot{x}_{k}(t)=A x_{k}(t)+A_{h} x_{k}\left(t-d_{k}(t)\right)+b u_{k}(t)+v_{k}(t), \\
& y_{k}(t)=x_{k}(t)+\omega_{k}(t),
\end{aligned}
$$

where the state delayed system with time varying delay which is nonrepeatable is analyzed. Random noises which are nonrepeatable are utilized to demonstrate the robustness of the system, and the initial input is zero. It is shown as $A=1 / 2$, 

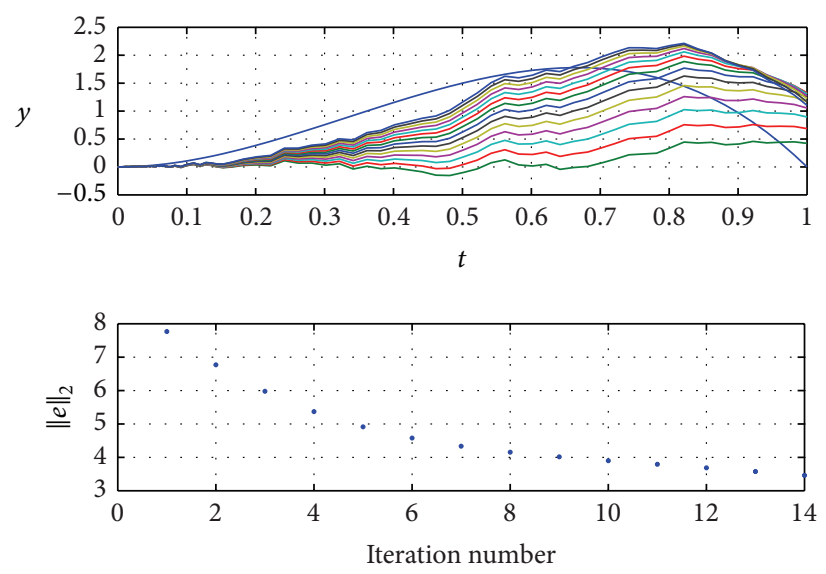

FIGURE 4: The simulation results of the ILC scheme (45) and (2).

$A_{h}=-1$, and $b=7 / 10$. Both $v_{k}(t)$ and $\omega_{k}(t)$ are random noises. Besides, let the learning gains be $K_{1}=4 / 5, K_{2}=1 / 10$, and $K_{3}=1 / 2$ are controller gains, $d_{k}(t)$ is the time varying delay which is nonrepeatable, and the upper bound of $d_{k}(t)$ is $h$, where $h=0.1$ denotes the upper bound of the time varying delay. All the parameters and the learning gains of the system satisfy the convergence condition in Theorem 3 at the beginning of Section $3(t=-0.0112)$; however, in the case of unknown system parameters the learning gains are achieved by small gain theorem. The initial control input $u_{0}(t)=0$ and the reference is $y_{d}(t)=12 t^{2}(1-t)$. The simulation results are shown in Figure 4, where the plots of output $y_{k}(t), k=$ $1,2,3, \ldots, 10$, and the 2 -norm of tracking errors are shown in it. It can be seen from Figure 4 that the convergence process is not strictly a monotonic one influenced by the random noise, and when $k=6,\left\|y_{d}(t)-y_{6}(t)\right\|_{2}=4.6$.

As one of the important performances of a system, the analysis of the convergence speed is indispensable. Unlike the LTI cases, it is verified from a number of simulation results that all the learning gains influence the fact of convergence and the convergence speed of the ILC scheme. A relatively faster convergence speed can be expected for larger $K_{1}$ and for smaller $K_{2}$ and $K_{3}$. Besides, what is to be highlighted is that the convergence conditions must be satisfied for the analysis of the convergence speed above. A low-pass filter such as $1 /((1 / 20) s+1)$ can be cascaded after the system output to cancel out the high frequency noises and reduce the tracking errors, which can further lead to an instrumental variable method of the system identification [36].

Lastly, given parameters of $A=1 / 2, A_{h}=-1, b=7 / 10$, and $C=1$, with the learning gains of $K_{1}=4 / 5, K_{2}=$ $1 / 10$, and $K_{3}=1 / 2$, the influences of delay and frequency to $|G e|$ are illustrated in Figure 5. It is shown that, in the low frequencies, a relatively faster convergence speed can be expected for larger time delay, while, in the high frequencies, the relatively faster convergence speed can be expected for smaller time delay.

5.3. Example 3. The purpose of identification in this paper is that the optimal controller is designed by the optimal

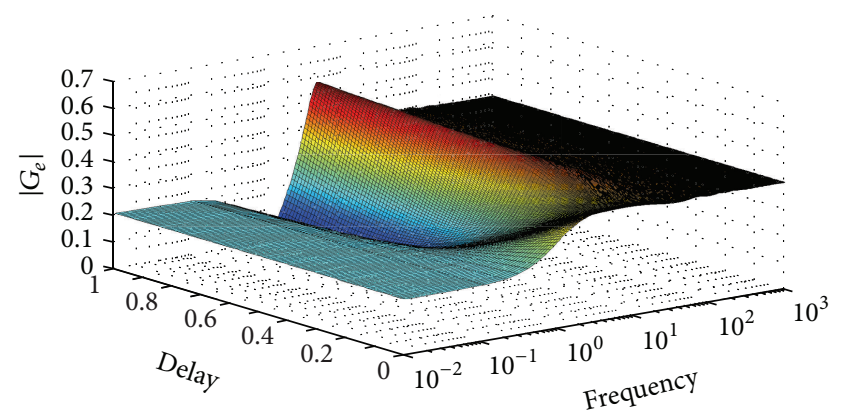

FIGURE 5: The relationships among frequency, delay, and convergence speed for the ILC scheme (45) and (2).

solutions of the identification by the assumption of the upper bound of time delay and system structure. The optimal controller is utilized in the time varying delay systems. Meanwhile, by the process of the ILC, the system parameters and the learning gains keep updating to be the optimal ones (based on the minimum average distance method).

Consider the system as

$$
\begin{aligned}
\dot{x}_{k}(t) & =A x_{k}(t)+A_{h} x_{k}(t-h)+B u_{k}(t), \\
y_{k}(t) & =C x_{k}(t) .
\end{aligned}
$$

Here, the parameters of the real system are defined as $A=0.5$, $A_{h}=-1, B=0.7$, and $C=1$. The time delay $h=0.1$. The parametric learning law is (2). The learning gains are $K_{1}=$ $4 / 5, K_{2}=1 / 10$, and $K_{3}=1 / 2$ and the sample time is 0.0001 . The convergence in the frequency domain is achieved as

$$
\begin{aligned}
& \left\|G_{e}(s)\right\|_{\infty} \\
& \quad=\left\|\left[I+G_{p}(s) K_{1}\right]^{-1}\left[I-G_{p}(s)\left(-K_{1}+K_{2} s+K_{3}\right)\right]\right\|_{\infty} \\
& \quad<\gamma<1,
\end{aligned}
$$

where $G_{p}(s)$ is the transformation of the real system.

The data information of the input and output is achieved in the workspace of MATLAB. The basic equations utilized are

$$
\begin{aligned}
y(t) & =\frac{1}{A} y^{\prime}(t)-\frac{A_{h}}{A} y(t-h)-\frac{C B}{A} u(t) \\
& =\left(y^{\prime}(t)-y(t-h)-u(t)\right)\left(\begin{array}{c}
\frac{1}{A} \\
\frac{A_{h}}{A} \\
\frac{C B}{A}
\end{array}\right) .
\end{aligned}
$$

Then the identified model can be gained by the method of identification which is introduced before, and all the solutions are shown in Table 1. By using the six groups of the data information in the workspace and by the minimum average distance method, the optimal identified parameters 
TABLE 1: Identification results.

\begin{tabular}{lcc}
\hline$A$ & $A_{h}$ & $B$ \\
\hline 0.5214 & -1.0203 & 0.6985 \\
0.5156 & -1.0143 & 0.6988 \\
0.5158 & -1.0146 & 0.6988 \\
0.5155 & -1.0142 & 0.6988 \\
0.5156 & -1.0143 & 0.6988 \\
0.5156 & -1.0144 & 0.6988 \\
\hline
\end{tabular}
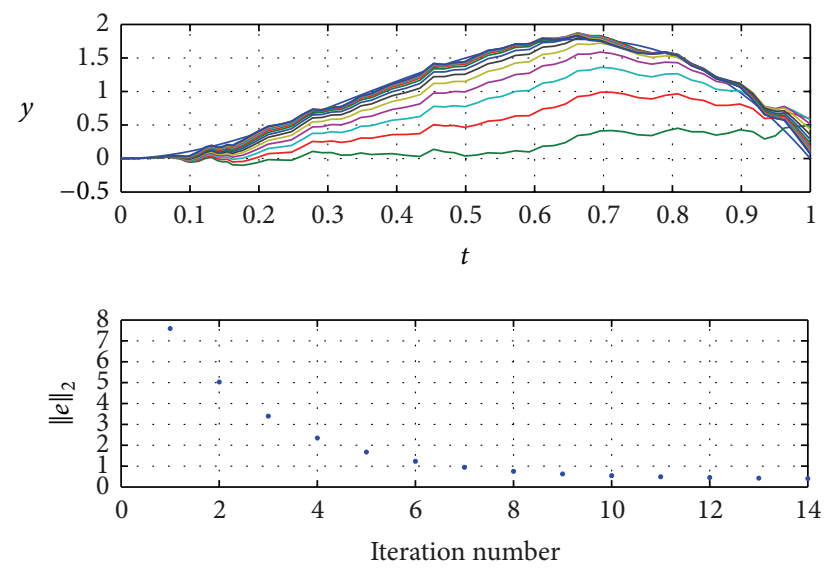

FIGURE 6: The optimal simulation results of the ILC scheme from (46), (2), and (48).

of $\bar{A}, \bar{A}_{h}$, and $\bar{B}$ are $0.516,-1.014$, and 0.699 , respectively. The identification errors are $0.016,0.014$, and 0.001 . By the identified values of parameters, the optimal controller can be designed which leads to the fastest convergence speed; that is, $\left|\bar{G}_{e}(s)\right|$ is minimized, where $K_{1}$ is fixed and $K_{2}$ and $K_{3}$ need to be designed. In this example, $K_{2}=0.5690$ and $K_{3}=0.2605$. The simulation results are shown in Figure 6. Compared to Figure 4, a much faster convergence speed can be achieved, which implies the efficiency of the proposed strategy.

Remark 6. The method of identification is efficient to identify the unknown parameters in time delay systems. The output tracking errors and parameter tracking errors can be achieved in the neighbourhood of 0.01. By the exact identified model, the optimal controller can be designed through the convergence condition to improve the convergence speed a lot. A low-pass filter can be cascaded after the output which further leads to an instrumental variable method of the system identification.

\section{Conclusions}

The convergence conditions of the MIMO time delay system are achieved in the time domain using LMI approach and $H_{\infty}$ method. The delay is time varying which is nonrepeatable. The robust parametric ILC scheme is applied while the learning gains in the learning law are designed to be the optimal ones by the solutions of the LMIs in the convergence condition. For improving the system performance, the identification is necessary to be utilized to determine the unknown parameters exactly. The convergence speed is analyzed in the paper. All the learning gains and the time delay influence the convergence speed. A relatively faster convergence speed can be expected for larger $K_{1}$ and for smaller $K_{2}$ and $K_{3}$. The relationship among the convergence speed, frequency, and the time delay is that, in the low frequencies, a relatively faster convergence speed can be expected for larger time delay, while, in the high frequencies, the relatively faster convergence speed can be expected for smaller time delay. From the convergence condition, it is known that the parameters in the system are key factors in the analysis. An identification method is applied to identify the parameters when those are unknown. The minimum average distance method is applied to gain the optimal solution of the parameters in all the identified solutions. The controller can be designed by the exact parameters to be the optimal one which improves the convergence speed and reduces the conservatism. The simulations by using MATLAB validate the conclusions in this paper.

Our future works include the robust ILC of nonlinear systems and the development of novel nonlinear ILC schemes.

\section{Conflict of Interests}

The authors declare that there is no conflict of interests regarding the publication of this paper.

\section{Acknowledgment}

This work is supported by the National Natural Science Foundation of China (61104009 and 61374101).

\section{References}

[1] M. Uchiyama, "Formulation of high-speed motion of a mechanical arm by trial," Transactions of SICE, vol. 14, no. 6, pp. 706712, 1978

[2] S. Arimoto, S. Kawamura, and F. Miyazaki, "Bettering operation of robots by learning," Journal of Robotic Systems, vol. 1, no. 2, pp. 123-140, 1984.

[3] A. Tayebi, "Analysis of two particular iterative learning control schemes in frequency and time domains," Automatica, vol. 43, no. 9, pp. 1565-1572, 2007.

[4] C. K. Yin, J. X. Xu, and Z. S. Hou, "An ILC scheme for a class of nonlinear continuous-time systems with time-iteration-varying parameters subject to second-order internal model," Asian Journal of Control, vol. 13, no. 1, pp. 126-135, 2011.

[5] H.-S. Ahn, L.-M. Kevin, and Y.-Q. Chen, Iterative Learning Controlrobustness and Monotonic Convergence for Interval Systems, Communications and Control Engineering, Springer, 2007.

[6] Y. Chen, Z. Gong, and C. Wen, "Analysis of a high-order iterative learning control algorithm for uncertain nonlinear systems with state delays," Automatica, vol. 34, no. 3, pp. 345-353, 1998.

[7] Z. Bien and K. M. Huh, "Higher-order iterative learning control algorithm," IEE Proceedings D: Control Theory and Applications, vol. 136, no. 3, pp. 105-112, 1989. 
[8] J. X. Xu, K. Abidi, X. L. Niu, and D. Q. Huang, "Sampleddata iterative learning control for a piezoelectric motor," in Proceedings of the 21st IEEE International Symposium on Industrial Electronics (ISIE '12), pp. 899-904, Hangzhou, China, May 2012.

[9] S. Dominik and A. Harald, "Robust iterative learning control for nonlinear systems with measurement disturbances," in Proceedings of the American Control Conference, pp. 5484-5489, Montreal, Canada, 2012.

[10] Y. Chen, C. Wen, and M.-X. Sun, "A robust high-order P-type iterative learning controller using current iteration tracking error," International Journal of Control, vol. 68, no. 2, pp. 331342, 1997.

[11] J. Xu and Y. Tan, "On the P-type and Newton-type ILC schemes for dynamic systems with non-affine-in-input factors," Automatica, vol. 38, no. 8, pp. 1237-1242, 2002.

[12] J.-X. Xu and Y. Tan, Linear a nd Nonlinear Iterative Learning Control, vol. 291 of Lecture Notes in Control and Information Sciences, Springer, Berlin, Germany, 2003.

[13] L.-J. Zhang, D.-Z. Cheng, and J.-B. Liu, "Stabilization of switched linear systems," Asian Journal of Control, vol. 5, no. 4, pp. 476-483, 2003.

[14] X. Bu, F. Yu, Z. Hou, and F. Wang, "Iterative learning control for a class of linear discrete-time switched systems," Acta Automatica Sinica, vol. 39, no. 9, pp. 1564-1569, 2013.

[15] M. X. Sun and Q. Z. Yan, "Error tracking of iterative learning control systems," Acta Automatica Sinica, vol. 39, no. 3, pp. 251262, 2013.

[16] W. H. Moase and C. Manzie, "Fast extremum-seeking for Wiener-Hammerstein plants," Automatica, vol. 48, no. 10, pp. 2433-2443, 2012.

[17] D. Meng, Y. Jia, J. Du, and J. Zhang, “On iterative learning algorithms for the formation control of nonlinear multi-agent systems," Automatica, vol. 50, no. 1, pp. 291-295, 2014.

[18] K. Delchev, "Iterative learning control for nonlinear systems: a bounded-error algorithm," Asian Journal of Control, vol. 15, no. 2, pp. 453-460, 2013.

[19] R. H. Chi, Z. S. Hou, S. T. Jin, and D. W. Wang, "Discrete-time adaptive ILC for non-parametric uncertain nonlinear systems with iteration-varying trajectory and random initial condition," Asian Journal of Control, vol. 15, no. 2, pp. 562-570, 2013.

[20] D. Shen and H. Chen, "A Kiefer-Wolfowitz algorithm based iterative learning control for Hammerstein-WIEner systems," Asian Journal of Control, vol. 14, no. 4, pp. 1070-1083, 2012.

[21] X. Ruan, Z. Z. Bien, and Q. Wang, "Convergence properties of iterative learning control processes in the sense of the LebesgueP norm," Asian Journal of Control, vol. 14, no. 4, pp. 1095-1107, 2012.

[22] X.-H. Bu, Z.-S. Hou, F.-S. Yu, and Z.-Y. Fu, "Iterative learning control for a class of non-linear switched systems," IET Control Theory \& Applications, vol. 7, no. 3, pp. 470-481, 2013.

[23] H. Sun, Z. Hou, and D. Li, "Coordinated iterative learning control schemes for train trajectory tracking with overspeed protection," IEEE Transactions on Automation Science and Engineering, vol. 10, no. 2, pp. 323-333, 2013.

[24] S.-P. Yang, J.-X. Xu, D.-Q. Huang, and Y. Tan, “Optimal iterative learning control design for multi-agent systems consensus tracking," Systems \& Control Letters, vol. 69, pp. 80-89, 2014.

[25] B. Kim, T. Lee, Y. Kim, and H. Ahn, "Iterative learning control for spatially interconnected systems," Applied Mathematics and Computation, vol. 237, pp. 438-445, 2014.
[26] Y. Song, Y. Li, X. Wang, X. Ma, and J. H. Ruan, "An improved reinforcement learning algorithm for cooperative behaviors of mobile robots," Journal of Control Science and Engineering, vol. 2014, Article ID 270548, 8 pages, 2014.

[27] J. Peng and Y. Liu, "Adaptive robust quadratic stabilization tracking control for robotic system with uncertainties and external disturbances," Journal of Control Science and Engineering, vol. 2014, Article ID 715250, 10 pages, 2014.

[28] H. Wu, "Decentralised adaptive robust control of uncertain large-scale non-linear dynamical systems with time-varying delays," IET Control Theory \& Applications, vol. 6, no. 5, pp. 629640, 2012.

[29] A. R. Fioravanti, C. Bonnet, and S. Niculescu, "A numerical method for stability windows and unstable root-locus calculation for linear fractional time-delay systems," Automatica, vol. 48, no. 11, pp. 2824-2830, 2012.

[30] C.-Y. Kao, "On stability of discrete-time LTI systems with varying time delays," IEEE Transactions on Automatic Control, vol. 57, no. 5, pp. 1243-1248, 2012.

[31] H.-B. Zhang, H. Zhong, and C.-Y. Dang, "Delay-dependent decentralized $H_{\infty}$ filtering for discrete-time nonlinear interconnected systems with time-varying delay based on the T-S fuzzy model," IEEE Transactions on Fuzzy Systems, vol. 20, no. 3, pp. 431-443, 2012.

[32] J. Doyle, "Analysis of feedback systems with structured uncertainties," Control Theory and Applications, vol. 129, no. 6, pp. 242-250, 1982.

[33] X. Jin and J. Xu, "Iterative learning control for output-constrained systems with both parametric and nonparametric uncertainties," Automatica, vol. 49, no. 8, pp. 2508-2516, 2013.

[34] B. Xu, X. Huang, D. Wang, and F. Sun, "Dynamic surface control of constrained hypersonic flight models with parameter estimation and actuator compensation," Asian Journal of Control, vol. 16, no. 1, pp. 162-174, 2014.

[35] D. Meng and Y. Jia, "Anticipatory approach to design robust iterative learning control for uncertain time-delay systems," Asian Journal of Control, vol. 13, no. 1, pp. 38-53, 2011.

[36] F. Le, I. Markovsky, C. T. Freeman, and E. Rogers, "Identification of electrically stimulated muscle models of stroke patients," Control Engineering Practice, vol. 18, no. 4, pp. 396-407, 2010.

[37] S. Toshiharu, "Identification of linear continuous-time systems based on iterative learning control," in Recent Advances in Learning and Control, pp. 205-218, 2008.

[38] F. Atsushi and O. Shinsuke, "Parameter identification of continuous-time systems using iterative learning control," International Journal of Control, Automation and Systems, vol. 9, no. 2, pp. 203-210, 2011.

[39] M. C. Campi, T. Sugie, and F. Sakai, "An iterative identification method for linear continuous-time systems," IEEE Transactions on Automatic Control, vol. 53, no. 7, pp. 1661-1669, 2008.

[40] L. Liu, K. K. Tan, and T. H. Lee, "SVD-based accurate identification and compensation of the coupling hysteresis and creep dynamics in piezoelectric actuators," Asian Journal of Control, vol. 16, no. 1, pp. 59-69, 2014.

[41] F. Sakai and T. Sugie, "An identification method for MIMO continuous-time systems via iterative learning control concepts," Asian Journal of Control, vol. 13, no. 1, pp. 64-74, 2011.

[42] J. Bendtsen and K. Trangbaek, "Closed-loop identification for control of linear parameter varying systems," Asian Journal of Control, vol. 16, no. 1, pp. 40-49, 2014. 
[43] Z.-S. Hou and S.-T. Jin, "A novel data-driven control approach for a class of discrete-time nonlinear systems," IEEE Transactions on Control Systems Technology, vol. 19, no. 6, pp. 1549-1558, 2011.

[44] T.-Y. Doh, J. R. Ryoo, and D. E. Chang, "Robust iterative learning controller design using the performance weighting function of feedback control systems," International Journal of Control, Automation and Systems, vol. 12, no. 1, pp. 63-70, 2014.

[45] O. Gaye, L. Autrique, Y. Orlov, E. Moulay, S. Brémond, and R. Nouailletas, " $H_{\infty}$ stabilization of the current profile in tokamak plasmas via an LMI approach," Automatica, vol. 49, no. 9, pp. 2795-2804, 2013.

[46] X.-Z. Jin, G.-H. Yang, X.-H. Chang, and W.-W. Che, "Robust faulttolerant $H_{\infty}$ control with adaptive compensation," Acta Automatica Sinca, vol. 39, no. 1, pp. 31-42, 2013.

[47] Y.-Q. Ye, D.-W. Wang, B. Zhang, and Y.-G. Wang, "Simple LMI based learning control design," Asian Journal of Control, vol. 11, no. 1, pp. 74-77, 2009.

[48] D. Meng, Y. Jia, J. Du, and F. Yu, "Delay-dependent conditions for monotonic convergence of uncertain ILC systems: an LMI approach," in Proceedings of the 49th IEEE Conference on Decision and Control (CDC '10), pp. 6955-6960, Atlanta, Ga, USA, December 2010.

[49] L. Zhai, G.-H. Tian, F.-Y. Zhou, and Y. Li, “The robust iterative learning control of networked control systems with varying references," in Proceeding of the 25th Chinese Control and Decision Conference (CCDC '13), pp. 19-24, Guiyang, China, May 2013.

[50] L. Zhai, G. Tian, F. Zhou, and Y. Li, "A frequency analysis of time delayed iterative learning control system," in Proceedings of the 32nd Chinese Control Conference (CCC '13), pp. 256-261, Xian, China, July 2013.

[51] M. Wu, Y. He, and J.-H. She, Stability Analysis and Robust Control of Time-Delay Systems, Springer, Berlin, Germany, 2010.

[52] M. Wu, Y. He, J.-H. She, and G.-P. Liu, "Delay-dependent criteria for robust stability of time-varying delay systems," Automatica, vol. 40, no. 8, pp. 1435-1439, 2004. 

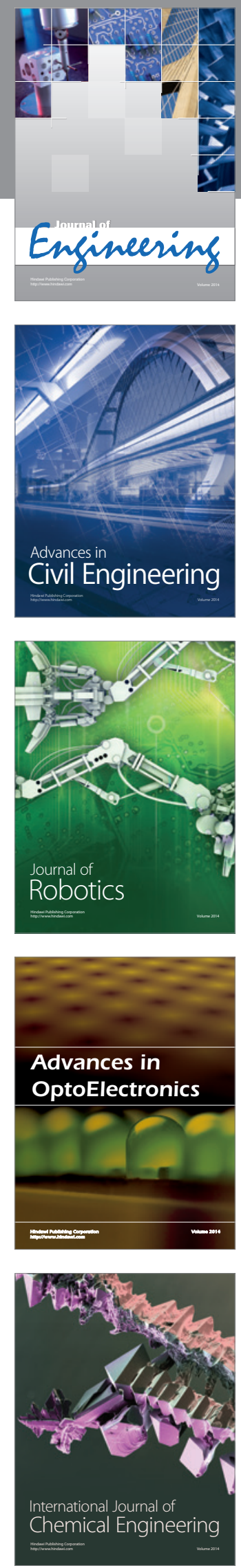

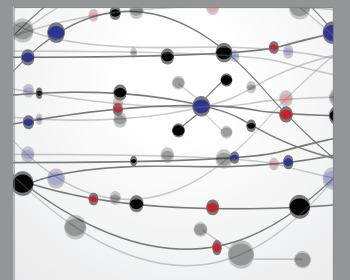

The Scientific World Journal
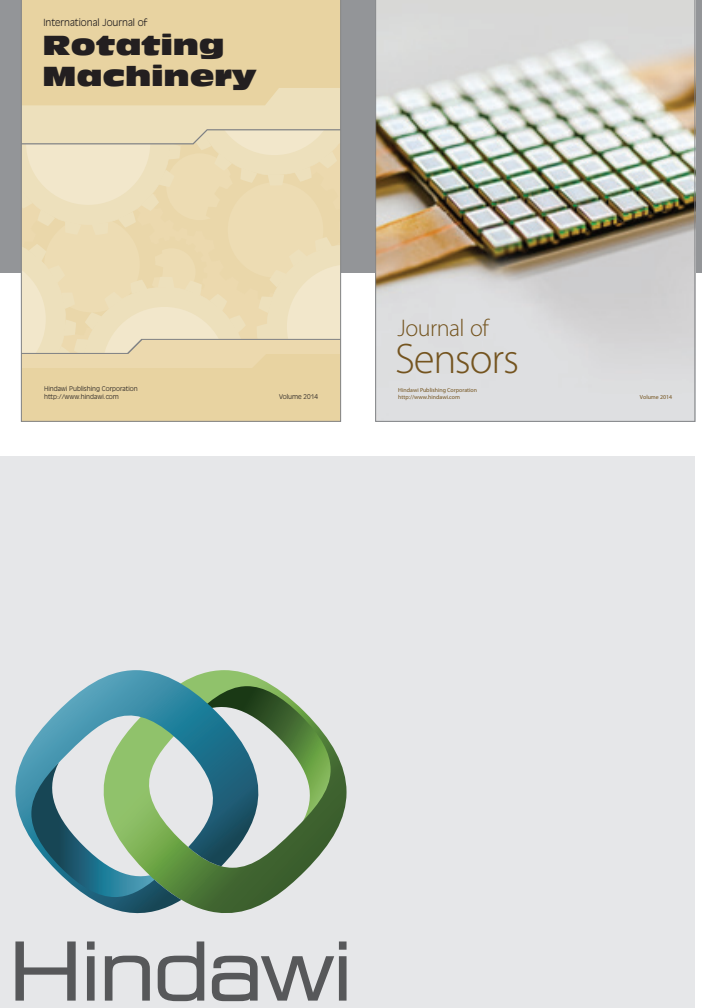

Submit your manuscripts at http://www.hindawi.com
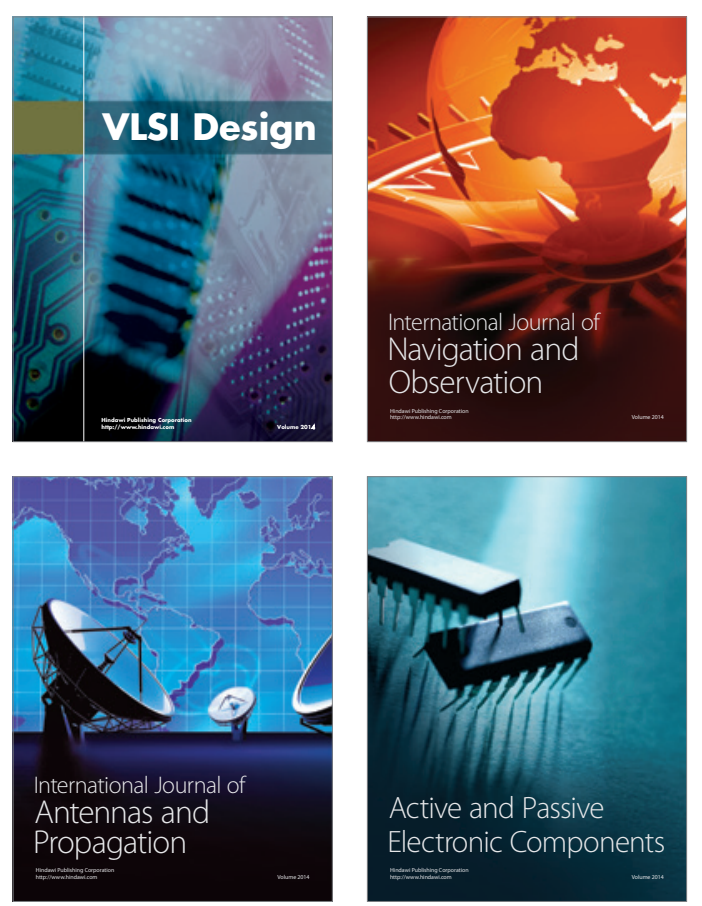
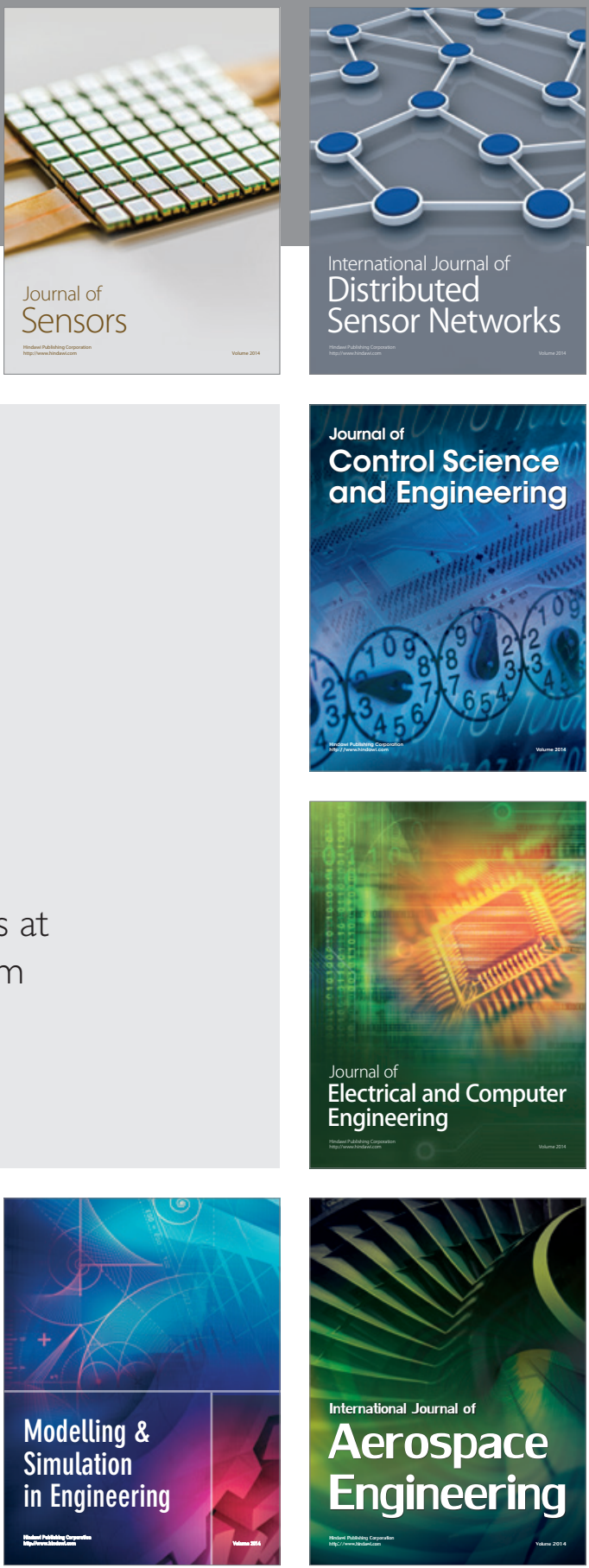

Journal of

Control Science

and Engineering
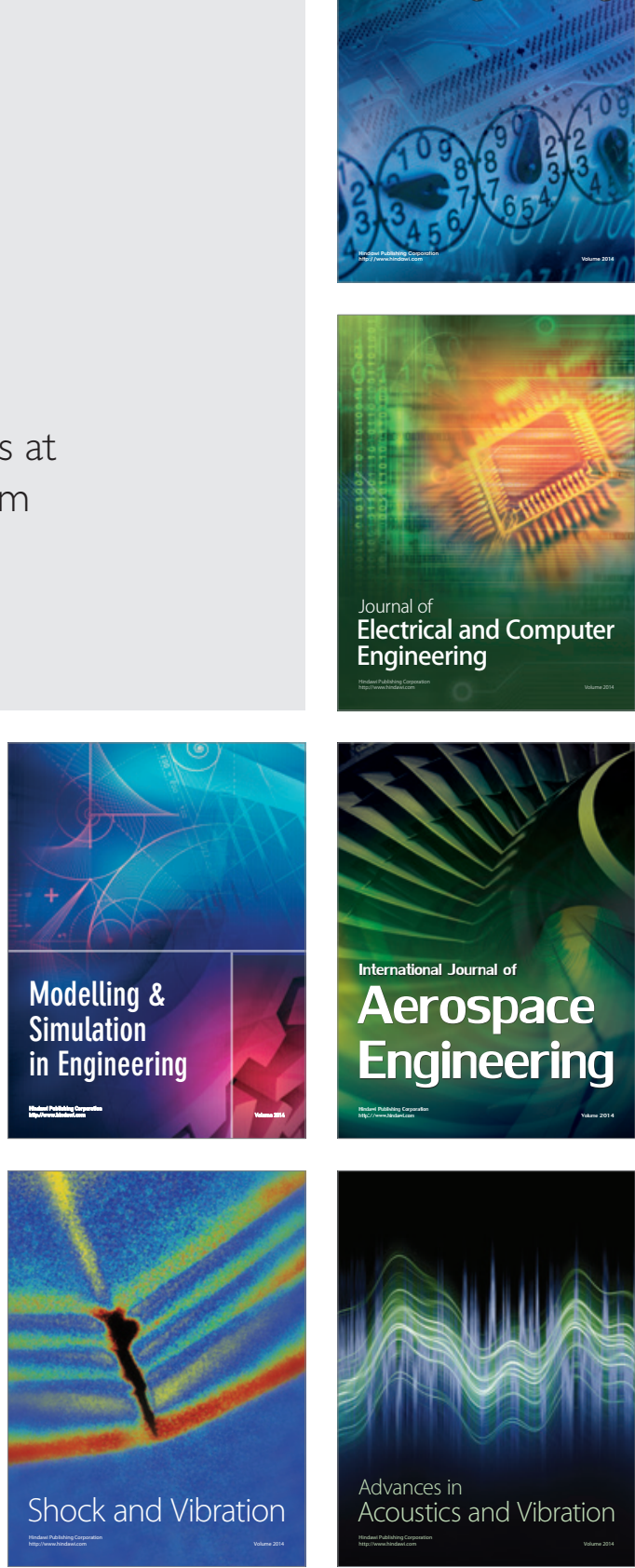